\title{
The physician's role in selecting a factor replacement therapy
}

S. W. PIPE

Pediatric Hemophilia and Coagulation Disorders Program, University of Michigan, Women's Hospital, Ann Arbor, MI, USA

Summary. Over the past 20 years, transmissions of human immunodeficiency virus (HIV), hepatitis B virus or hepatitis $\mathrm{C}$ virus have been virtually eliminated from plasma-derived or recombinant therapy in the USA, a record that can be largely attributed to the use of effective screening and inactivation technologies for known pathogens. The next significant threat will likely come from the emergence of a new, blood-borne infectious disease, perhaps one transmitted by a non-lipid-enveloped virus or prion, for which current inactivation methods are ineffective. Following the HIV crisis of the 1980s, government, patient advocacy groups, medical and scientific communities and the manufacturers of clotting therapies can learn from the past and approach potential threats from emerging pathogens in a proactive and productive manner. For clinicians, this includes actively engaging patients in a dialogue about all the factors that may influence their choice of clotting factor therapies, including emerging pathogens, patient convenience, consistency and reliability of supply, relative cost/benefit ratios, reimbursement issues (where applicable), patient preference and brand loyalty. It is our obligation as healthcare providers to understand potential risks and help make proactive decisions with our patients, decisions that often must be made in an environment of scientific uncertainty. Threats from infectious agents that were once deemed theoretical can, and often do, ultimately become real, with serious implications for morbidity and mortality.

Keywords: haemophilia, pathogens, plasma, recombinant, therapy

\section{Introduction}

The principle of evidence-based medicine promotes the judicious and conscientious use of the current best evidence when making healthcare decisions for individual patients. While this strategy, popularized in the early 1990s, is frequently useful, it is clearly of limited benefit in the context of emerging infectious diseases. By the time concrete evidence of an emerging infectious agent is available, it is often too late to prevent infection in the most susceptible populations. Proactive decision-making processes regarding the safety of our blood supply cannot rely solely on an evidenced-based approach. Rather we can take the lessons of the past and, using our current knowledge of disease causing agents, extrapolate potential risks in order to better formulate effective healthcare policies.

Correspondence: Steven W. Pipe, MD, Pediatric Hemophilia and Coagulation Disorders Program, University of Michigan, Women's Hospital, 1500 East Medical Center Drive, Ann Arbor, MI 48109, USA.

Tel.: 1734647 2893; fax: 1734936 7083;

e-mail: ummdswp@med.umich.edu

\section{Learning from the past}

Within the USA, in the years immediately preceding the human immunodeficiency virus (HIV) epidemic, medical and scientific communities, government agencies and the blood therapies industry operated in an environment of shared responsibility to ensure the safety of the blood supply. Through the exercise of regulatory authority, established standards for plasma collection, product manufacturing and licensing, blood surveillance and fundamental research efforts, the system worked effectively to supply the nation with necessary blood and blood products that checked for most human safety threats. The weakness of this system was deemed to be its inability to deal with a new threat that was characterized by substantial uncertainty. This was the subject of a report from the Institute of Medicine commissioned by the Department of Health and Human Services [1]. From the first description of cases of HIV in haemophilia patients in 1982 to the development of a serum test for HIV in 1985, policy-making was extremely difficult as much more was unknown than known. During that time, evidence of the risk of acquired immunodeficiency syndrome (AIDS) was 
downplayed, even regarded as 'theoretical', and time was lost while evaluating the estimates of the substantial costs of safeguards. The high regard for the efficacy of antihaemophilic factor concentrates for treating a devastating disease like haemophilia, as well as scepticism that AIDS was transmitted through blood products, led to a lack of specific recommendations about blood product use from patient groups and physicians treating individuals with haemophilia. In turn, rather than inform patients about risks and benefits, physicians tended to decide for themselves what was best so as not to burden patients with difficult decisions about treatment options [2].

In defence of these stakeholders, initially there was little or no reliable evidence of HIV [and later hepatitis $\mathrm{C}$ virus (HCV) transmission] via plasmabased therapies. As the 1980s proceeded, however, research showing that these diseases were, in fact, transmitted through blood products began to increase and come to the attention of all involved with the haemophilia community.

While the transmission pathways and disease processes for HIV and HCV are now well understood, uncertainty remains about the potential for variant Creutzfeldt-Jakob disease (vCJD) and other emerging pathogens to harm patients with haemophilia. The clear message is that it is our obligation as healthcare providers to help make proactive decisions with our patients, and that often these must be made in an environment of scientific uncertainty. Threats from infectious agents that were once deemed theoretical can, and often do, ultimately become real, with serious implications for morbidity and mortality.

\section{Safety concerns: pathogen risks and inactivation efforts}

The current safety of haemophilia therapies can largely be attributed to the use of effective screening and testing technologies available for HIV, Hepatitis $B$ virus (HBV) or HCV. No seroconversions to HIV, HBV or HCV have been reported with any of the FVIII products currently marketed in the USA [3]. The real challenge, however, is presented by the likely emergence of a new, blood-borne infectious agent. For this, we rely on inactivation measures.

For the purposes of discussing inactivation measures, infectious agents can be classified in three categories:

1 Lipid-enveloped viruses, for example, HBV, HCV and HIV
2 Non-lipid-enveloped viruses, for example, parvovirus B19 (PVB19) and hepatitis A (HAV)

3 Disease-causing prions, for example, CJD and vCJD

\section{Lipid-enveloped viruses}

Lipid-enveloped viruses have a protective fatty membrane, or envelope; if this membrane is destroyed the enclosed virus is also destroyed. There is very good evidence that the lipid-enveloped viruses are effectively inactivated with current technologies [4-6]. All licensed processors of plasma therapies and recombinant therapies made with human and animal protein additives use validated methods to both detect and eliminate HBV, HCV, HIV, and the other lipid-enveloped viruses for which screening and inactivation methods have been developed [7]. West Nile virus is another lipid-enveloped virus for which effective screening methods exist; for example, in the period JanuaryNovember 15, 2005, 382 presumptively viremic blood donors were successfully detected in the USA [8].

\section{Non-lipid-enveloped viruses}

As the term would indicate, non-lipid-enveloped viruses lack a protective enclosure. The lack of an envelope makes it difficult to target these viruses for inactivation. In addition, the physical and chemical conditions created by vapour/heat, solvent/detergent and gamma irradiation technologies adequate for inactivation of non-lipid-enveloped viruses may denature the factor VIII (FVIII) protein [9]. The small size of some non-lipid-enveloped viruses, such as PVB19, requires filter sizes generally not practical for the successful large-scale filtration of commercial blood products [10].

\section{Parvovirus B19}

Human PVB19 is a widespread non-lipid-enveloped virus that causes the childhood illness fifth disease. Community-acquired exposure and seroconversion to this virus is common and occurs quite early in the lives of most people. PVB19 shares the hallmark of other non-lipid-enveloped viruses: resistance to inactivation technologies. PVB19 is of particular concern to the haemophilia community because of reports that it has been found in factor concentrates. A prospective study published in 1997 by Santagostino et al. [11] indicated that very high temperatures applied to lyophilized factor concentrates did not prevent the transmission of PVB19 to patients with haemophilia. 
Soucie et al. [12] evaluated the risk of PVB19 transmission as a result of treatment with recombinant antihaemophilic factor. To compare the seroprevalence of PVB19 antibodies in 2- to 7-year-old males with haemophilia, 798 subjects were grouped by their exposure to types of factor VIII or IX concentrates: those who had received only plasmaderived therapies, only recombinant therapies both recombinant and plasma-derived therapies, or no antihaemophilic factor at all (control group). The study found that the prevalence of seropositivity was higher in both the plasma-derived only and recombinant and plasma-derived therapies groups than in the control and recombinant-only groups [12].

The results of this study illustrate the potential for non-lipid-enveloped viruses to persist in blood and blood-based therapies. In 2001, processors of plasma-derived factor therapies instituted the use of nucleic acid amplification technology (NAT) to screen plasma and adopted a voluntary industry standard for the management of PVB19. Nonetheless, Soucie et al. [12] recommended the development of effective virus inactivation techniques for parvovirus and other non-enveloped viruses that have yet to be identified or may emerge in the future.

\section{Disease-causing prions}

As with PVB19 and other non-lipid-enveloped viruses, current inactivation methods are relatively ineffective against prions. Prions are soluble cellular protein particles $\left(\operatorname{PrP}^{\mathrm{C}}\right)$ that lack nucleic acid and do not depend on genes or other factors for transmission. As discussed elsewhere in this supplement, transmissible spongiform encephalopathies (TSEs) such as the sporadic and variant forms of CJD are characterized by the accumulation of an abnormal form of this protein particle $\left(\mathrm{PrP}^{\mathrm{Sc}}\right)$ in the brain.

Because prions lack nucleic acid, standard NAT testing cannot be used to detect vCJD and other TSEs. Also, the presence of abnormal prions does not trigger a measurable host immune response, making standard enzyme-linked diagnostic tests that measure antibodies, such as those used for AIDS, useless. New, hopefully more effective screening methods are currently in development.

During the disease's incubation period, estimated to be upwards of 40 years, infected individuals appear clinically healthy. Without a screening method for vCJD, donations by infected individuals will continue to pose a threat to patients using bloodderived therapies.

\section{Opportunities for discussions}

Most patients become aware of the worldwide emergence of air- and blood-borne pathogens through articles and news broadcasts in the popular press. Without the benefit of broad-based medical knowledge for context, patients may become unduly alarmed about the risks these infectious agents pose. Haemophilia treaters have an opportunity to be proactive in addressing their patients' fears about these diseases and the particular health threats that blood-borne infectious agents might pose. To be effective, practitioners must themselves be informed about these new infectious agents and the relative safety of the available therapeutic options.

The point of these discussions is to address patients' fear and anxiety with regard to these new infectious diseases and to enhance patient trust in both haemophilia treaters and their therapeutic options. Fear is unavoidable if the potential threats of emerging pathogens are not met with an informed and appropriate response.

\section{Discussing the risk of transmission via factor therapies}

Haemophilia treaters must be cognizant of and acknowledge the risk that emerging pathogens present their especially vulnerable patients. The majority of practitioners are cautiously confident about the safety of current plasma-derived clotting factors and recombinant therapies $[13,14]$. Their optimism is the result of $>20$ years of access to blood therapies free of HIV and $>10$ years of access to therapies free of HCV.

Today paediatric haemophilia treaters, for example, see few if any HIV- or HCV-infected patients [15]. As a result, it is unlikely that these practitioners engage in regular conversations with their patients regarding emerging pathogens or the safety of the blood supply. Haemophilia consumer or advocacy groups have shared the responsibility for educating patients about these risks. However, in order to offer their patients with haemophilia the best possible care and to maintain trust, clinicians themselves must address their individual patients' needs and concerns with up-to-date information.

\section{Current treatment guidelines}

In recent years, patient advocacy groups and haemophilia treaters' organizations have provided information, recommendations and guidelines to help educate practitioners and others in the haemophilia community about the issue of the safety of the blood 
supply. Recommendations for proactive therapeutic measures have been implemented in the past regarding clotting factor concentrates. Within the USA and the EU, most patients with haemophilia have switched from plasma-derived to recombinant therapies since the first recombinant FVIII was approved in 1992 $[16,17]$. The majority of Canadian haemophilia patients were converted to recombinant FVIII in 1994 [18]. This decision was made by the Canadian Blood Agency at the advice of the Association of Hemophilia Clinic Directors of Canada who considered recombinant FVIII to be the safest replacement therapy available. Further recommendations have followed regarding vulnerabilities that may remain in the recombinant therapies owing to the addition of human or animal proteins during processing and final formulation. For example, in their 1999 update of the 1995 clinical practice guidelines for patients with haemophilia and von Willebrand disease, the Association of Hemophilia Clinic Directors of Canada stated that, "until methods for total viral removal or inactivation are available, alternative methods of cell culture and stabilization of recombinant clotting factor concentrates should be sought to avoid the need for plasma-derived human albumin that's currently in use' [19]. Similarly, in November 2003, the Medical and Scientific Advisory Council (MASAC) of the US-based National Hemophilia Foundation (NHF) published Recommendation 151, advising manufacturers that 'all efforts should be made to remove human albumin from recombinant FVIII products' [3]. The first recombinant factor replacement therapy processed without the addition of any human or animal plasma proteins and albumin was approved by the US Food and Drug Administration (FDA) earlier that year, in July 2003. The UK Department of Health began a rolling phase-out of plasma-derived factor replacement therapies [20] and promoted the use of recombinant therapies that do not contain human or animal protein additives as the firstline choice for adults with haemophilia A and B; children under the age of 16 had previously been transferred to recombinant products following a 1998 provision. These expanded measures were taken as a precaution against possible $\mathrm{vCJD}$ transmission through blood and blood products. These guidelines indicate the proactive measures some organizations are taking to protect their communities from bloodborne infectious agents.

\section{Additional factor replacement considerations}

The primary goal of any haemophilia practitioner is to enable one's patients to live as actively and productively as possible. Selecting the appropriate factor replacement therapy for each patient can be very complex. Safety and efficacy are first and foremost considerations in choosing a haemophilia therapy. Inhibitor risk is also an important concern. Other considerations include patient convenience, and issues related to the consistency and reliability of supply for any particular therapy $[14,21]$. The relative cost/benefit ratio may also be a consideration, depending on which therapy is chosen $[13,22,23]$. In the USA, policies regarding reimbursement for therapies can differ from state to state, which can cause confusion and consternation for patients and providers alike. Last, but not least, it is important to take patient preference and brand loyalty under consideration when choosing the appropriate factor therapy as some patients have been resistant to switching to new products $[22,24]$.

\section{Conclusion}

Underestimating what were seen as merely theoretical risks in the late 1970s and early 1980s ultimately resulted in the tragedy of HIV and HCV infection in patients with haemophilia worldwide. With the benefit of hindsight and the commitment of a proactive approach to emerging pathogens even in the face of scientific uncertainty, patients with haemophilia should hopefully never have to endure such a crisis again.

The most fundamental lesson learned in the past 25 years is that government, patient advocacy groups, medical and scientific communities and the manufacturers of clotting therapies all have an opportunity, and perhaps even an obligation, to approach potential threats from emerging pathogens in a proactive and productive manner. For clinicians, this includes actively engaging patients in a dialogue about emerging pathogens and the relative risks they might pose via available clotting factor therapies.

\section{References}

1 Committee to Study HIV Transmission Through Blood and Blood Products, Institute of Medicine. Leveton LB, Sox HC Jr, Stoto MA, eds. HIV and the Blood Supply: an Analysis for Crisis Decisionmaking. Washington, DC: National Academy Press, 1995; 1-17.

2 Stoto MA. The precautionary principle and emerging biological risks: lessons from swine flu and HIV in blood products. Public Health Rep 2002; 117: 546-52.

3 National Hemophilia Foundation. MASAC recommendations Concerning the Treatment of Hemophilia 
and Other Bleeding Disorders. MASAC Document no. 151, November 2003. Published online at http://www. hemophilia.org/research/masac/masac151.pdf. Accessed July 2004.

4 Chandra S, Groener A, Feldman F. Effectiveness of alternative treatments for reducing potential viral contaminants from plasma-derived products. Thromb Res 2002; 105: 391-400.

5 Hoots WK. History of plasma-product safety. Transfus Med Rev 2001; 15(Suppl. 1): 3-10.

6 Fischer G, Hoots WK, Abrams C. Viral reduction techniques: types and purpose. Transfus Med Rev 2001; 15(2 Suppl. 1): 27-39.

7 Centers for Disease Control and Prevention. CDC's Universal Data Collection project: blood safety. February 2002. Published online at http://www.cdc.gov/ ncbddd/hbd/blood_safety_facts.htm. Accessed March 2005.

8 Centers for Disease Control and Prevention, National Center for Infectious Diseases. 2005 West Nile virus viremic blood donor activity in the United States. Reported to the CDC as of November 15, 2005. Available at http://www.cdc.gov/ncidod/dvbid/ westnile/surv\&control05Maps_Viremic.htm. Accessed November 2005.

9 Miekka SI, Busby TF, Reid B, Pollock R, Ralston A, Drohan WN. New methods for inactivation of lipidenveloped and non-enveloped viruses. Haemophilia 1998; 4: 402-8.

10 Yokoyama T, Murai K, Murozuka T, Wakisaka A, Tanifuji M, Fujii $\mathrm{N}$ et al. Removal of small nonenveloped viruses by nanofiltration. Vox Sang 2004; 86: 225-9.

11 Santagostino E, Mannucci PM, Gringeri A et al. Transmission of parvovirus B19 by coagulation factor concentrates exposed to 100 degrees C heat after lyophilization. Transfusion 1997; 37: 517-22.

12 Soucie JM, Siwak EB, Hooper WC, Evatt BL, Hollinger FB and the Universal Data Collection Project Working Group. Human parvovirus B19 in young male patients with hemophilia A: associations with treatment product exposure and joint range-of-motion limitation. Transfusion 2004; 44: 1179-85.

13 Brown SA. Issues in making a therapeutic choice: recombinant and/or human-derived products. Haemophilia 2000; 6: 12-9.
14 Aledort LM. Making a therapeutic choice: human versus recombinant fractions - can we do it? Haemophilia 2001; 7(Suppl. 1): 1-3.

15 Soucie JM, Richardson LC, Evatt BL et al. Risk factors for infection with HBV and HCV in a large cohort of hemophiliac males. Transfusion 2001; 41: 338-43.

16 Chambost H, Ljung R. Changing pattern of care of boys with haemophilia in western European centres. Haemophilia 2005; 11: 92-9.

17 Giangrande P., Haemophilia B. Christmas disease. Expert Opin Pharmacother 2005; 6: 1517-24.

18 Giles AR, Rivard GE, Teitel J, Walker I. Surveillance for factor VIII inhibitor development in the Canadian Hemophilia A population following the widespread introduction of recombinant factor VIII replacement therapy. Transfus Sci 1998; 19: 139-48.

19 Association of Hemophilia Clinic Directors of Canada. Clinical practice guidelines: Hemophilia and von Willebrand's disease: 2. Management (Edition 2, Update 2). July 7, 1999. Published online at http:// www.ahcdc.ca/vWDManagement.html. Accessed April 2005.

20 United Kingdom Haemophilia Centre Doctors' Organisation on behalf of The Department of Health Forward Planning and Monitoring Group. Rollout of Recombinant Products for all Adult Haemophilia Patients in England: Report of 2003/2004. September 2004. Published online at http://www.dh.gov.uk/assetRoot/04/09/00/63/04090063.pdf. Accessed September 2005.

21 Farrugia A. Evolving perspectives in product safety for haemophilia. Haemophilia 2002; 8: 236-43.

22 Mauser-Bunschoten EP, Roosendaal G, van den Berg HM. Product choice and haemophilia treatment in the Netherlands. Haemophilia 2001; 7: 8.

23 Mantovani LG, Monzini MS, Mannucci PM, Scalone L, Villa M, Gringeri A. Differences between patients', physicians' and pharmacists' preferences for treatment products in haemophilia: a discrete choice experiment. Haemophilia 2005; 11: 589-97.

24 Miller R. The responsibility of separating truth from myth to patient and family. Haemophilia 2001; 7: 91-5. 